\title{
Article \\ Effects of Electron Correlation inside Disordered Crystals
}

\author{
Sergei P. Kruchinin ${ }^{1}\left(\mathbb{D}\right.$, Roberts I. Eglitis ${ }^{2, *}$, , Vitaliy P. Babak ${ }^{3}$, Iryna G. Vyshyvana ${ }^{4}$ and Stanislav P. Repetsky ${ }^{5,6}$
}

check for

updates

Citation: Kruchinin, S.P.; Eglitis, R.I.; Babak, V.P.; Vyshyvana, I.G.; Repetsky, S.P. Effects of Electron Correlation inside Disordered Crystals. Crystals 2022, 12, 237. https://doi.org/10.3390/ cryst12020237

Academic Editors: Christian Rodenbücher, Carsten Korte and Qingping Fang

Received: 30 December 2021

Accepted: 2 February 2022

Published: 9 February 2022

Publisher's Note: MDPI stays neutral with regard to jurisdictional claims in published maps and institutional affiliations.

Copyright: (C) 2022 by the authors. Licensee MDPI, Basel, Switzerland. This article is an open access article distributed under the terms and conditions of the Creative Commons Attribution (CC BY) license (https:// creativecommons.org/licenses/by/ $4.0 /)$.
1 Bogolyubov Institute for Theoretical Physics, 03143 Kyiv, Ukraine; sergeikruchinin@yahoo.com

2 Institute of Solid State Physics, University of Latvia, 8 Kengaraga Str., LV1063 Riga, Latvia

3 Institute of Engineering Thermophysics, NASU, 03057 Kyiv, Ukraine; vdoe@ukr.net

4 Institute of High Technologies, Taras Shevchenko Kyiv National University, 01601 Kyiv, Ukraine; i.vyshyvana@gmail.com

5 Department of Physical and Mathematical Sciences, National University of Kyiv-Mohyla Academy, 04070 Kyiv, Ukraine; srepetsky0208@gmail.com

6 G.V.Kurdyumov Institute for Metal Physics of the NAS of Ukraine, 03142 Kyiv, Ukraine

* Correspondence: rieglitis@gmail.com; Tel.: +371-26426703

\begin{abstract}
We propose a novel approach for characterising the electron spectrum of disordered crystals constructed from a Hamiltonian of electrons as well as phonons and a diagram approach for Green's function. The system's electronic states were modelled by means of the multi-band, tight-binding approach. The system's Hamiltonian is described based on the electron wave functions at the field of the atom nucleus. Our novel approach incorporates the long-range Coulomb interplay of electrons located in different lattice positions. Explicit interpretations of Green's functions are derived using a diagram method. Equations are obtained for the vertex components for the mass operators of the electron-electron as well aselectron-phonon interplays. A system of equations for the spectrum of elementary excitations in the crystal is obtained, in which the vertex components for the mass operators of electron-electron as well as electron-phonon interplays are renormalised. Thismakes it possible to perform numerical computationsfor the system's energy spectrum with a predetermined accuracy. In contrast to other approaches in which electron correlations are only taken into account in the limiting cases of an infinitely large and infinitesimal electron density, in this method, electron correlations are described in the general case of an arbitrary density. We obtained the cluster expansion of the density of states (DOS) of the disordered systems. We demonstrate that the addition of the electron-scattering mechanismsto the clusters is decreasing. This happens due to a growing number of positions in the cluster, which hang ontothe small parameter. The computing exactness is fixed by a small parameter for cluster expansion of Green's functions of electrons as well as phonons.
\end{abstract}

Keywords: new theory; electron correlation; electron spectrum; Hamiltonian of electrons; thermodynamic potential

\section{Introduction}

Breakthroughs in characterising the disordered systems are firmly connected with the evolution of the pseudopotential method [1]. The identical complication relates to apseudopotential method [1]. Due to a limited description of the pseudopotential, the difficulty of pseudopotential transferability remains. It is impossible to make use of the nuclear potentials set through the possessions of some systems in order to characterise another system. A fundamental breakthrough was accomplished duringthe research of the electronic structure, as well as properties of the system due to the application of the ultrasoft pseudopotentials developed by Vanderbilt [2,3] as well as the projector augmented waves theory developed by Blochl $[4,5]$. The previously mentioned theory was extended due to an application of the generalised gradient approximation (GGA) in the density functional theory (DFT) of the many-electron structures, refined in the papers [6-10]. In 
the augmented projector waves method, a wave function of valence states for the electron orbital (all-electron) is manifested due to the transformation via the pseudo orbital. The pseudo orbital expands into the pseudo fractional waves in the augment region. The all-electron orbital in the identical region is increased in size using the identical coefficients via partial waves, expressed by the Kohn-Sham equation. The term for the pseudoHamiltonian that we possess, in the equation with the pseudo wave function, is obtained by reducing the entire energy functional. From the previously mentioned equation, we can obtain the electron energetic spectrum wave functions as well as the value of the complete energy functional. The application of the method is very detailed, as described in [10] for the crystal electronic structure computed by means of the VASP computer codewell-known worldwide. By means of the cluster method's calculations, as well as the GAUSSIAN computer code, this method may be applied for the explanation of the electronic structure of molecules.

It is worth noting that in [11-19], recently straightforward productive computation approaches of the electronic structure, as well as properties of large molecules, have been suggested. The proposed approach is to build on the tight-binding representation and functional density theory, which incorporates long-range Coulomb interplay of electrons at different crystal lattice sites. Introduced approaches [6-19] are employed solely for the interpretation of pristine-ordered crystals and molecules. Effects related tothe electronic localised states, as well as lattice vibrations, happen at disordered crystals. These effects cannot be characterised using the model for a perfect crystal. On this subject, other methods are also developing.

Crucial accomplishments in illustration of the effects of the disordered systems are connected with the implementation of the tight-binding model for multi-electron scattering, which includes an estimation of the coherent potential. Beginning out of Slater's and Koster's contributions $[20,21]$, the tight-binding model was widely used in electronic structure computations and in the explanation of the ideal crystal characteristics. Computations for the electronic structure of the alloy are based upon using the self-consistentmethod, namely the Korringa-Kohn-Rostoker coherent-potential approximations, which are made in [22-24].

Calculations of the energetic spectrum of disordered crystals in our contribution wererefined by the theory on the basis of the function of Green. Electronic correlations in the crystal are expressed by means of themulti-band, tight-binding representation. Electronscattering actions on the nuclei potentialsof atoms of non-identical types, as well as on the oscillations of the crystal lattice, are included. Computations of two multiplications of Green's functionare derived from the temperature of Green's functions [25]. It makes use of a well-acceptedconnectionamong the spectral representation for two multiplications, as well as the temperature, of the Green's function [26]. Computation of the temperature for theGreen's functions for disordered crystal is formed on diagram technics, homologous to diagram technics for homogeneous systems [26]. The equation package of two multiplications of Green's functions of solids is obtained. Energetic spectrumcomputation accuracy is based upon re-normalisation of the vertex parts of the electron-electron as well as electron-phonon mass operators.

\section{Hamiltonian of an Electron-Phonon System of a Disordered Crystal}

The disordered system's Hamiltonian (disordered semiconductor or alloy)contains the Hamiltonian of electrons at the outer nucleus field, the Hamiltonian of electron-electron interplay, and the Hamilton of the nucleus, as well as the Hamiltonian of electron-nucleus interchange. The movement of an ion subsystem lessens to nucleus oscillations nearby thebalance position under the influence of the nucleus interplay force, as well as their indirect interplay via electrons. Using the Wannier representation, we can describe the system'sHamiltonian as follows [25]:

$$
H=H_{0}+H_{\text {int }}
$$


Consequently, the zero-order Hamiltonian is defined as:

$$
H_{0}=H_{e}^{(0)}+H_{p h}^{(0)}
$$

Itcomprisesthe electron's Hamiltonian inside the field of atom cores of the perfectlyarranged crystal:

$$
H_{e}^{(0)}=\sum_{\substack{n i \gamma \\ n^{\prime} i^{\prime} \gamma^{\prime}}} h_{n i \gamma, n^{\prime} i^{\prime} \gamma^{\prime}}^{(0)} a_{n i \gamma}^{+} a_{n^{\prime} i^{\prime} \gamma^{\prime}}
$$

Additionally, it comprises the phonon harmonic Hamiltonian of the movement for thecores of atoms in the ideally arranged crystal:

$$
H_{p h}^{(0)}=\sum_{n i \alpha} \frac{P_{n i \alpha}^{2}}{2 M_{i}}+\frac{1}{2} \sum_{\substack{n i \alpha \\ n^{\prime} i^{\prime} \alpha^{\prime}}} \Phi_{n i \alpha, n^{\prime} i^{\prime} \alpha^{\prime}}^{(0)} u_{n i \alpha} u_{n^{\prime} i^{\prime} \alpha^{\prime}}
$$

In this place, cores of the atoms are placed in the periodic lattice (namely, the unperturbed structure is periodically arranged, and it is without disorder). The character $n$ indicates the unit cell, $i$ indicates the $i$ th basis vector in the $n$th unit cell, and $\gamma$ indicates another quantum number for the orbital, inclusive of spin. Disorder will be introducedfor the sort of core at the specific lattice area, whichdoes not needto be periodic through theperturbed Hamiltonian term (view below). The character $h^{(0)}$ symbolises the "hopping integral" that links the relevant orbitals. For the phonon Hamiltonian, $n$ and $i$ are the same variables as previously, that is, the unit cell and basis site inside the unit cell. At the same time, $\alpha$ is the spatial direction $(x, y$, or $z)$. Namely, $P_{n i \alpha}$ describes the core momentum, $M_{i}$ describes themass of the core, $u_{n i \alpha}$ describes the variation of the core from the balance position of the lattice site, and $\Phi_{n i \alpha, n^{\prime} i^{\prime} \alpha^{\prime}}^{(0)}$ describes the consonant springconstant matrix. The interplay Hamiltonian in Equation (1) is the disturbance of the system caused by all, includingby our effects. It contains six parts:

$$
H_{\mathrm{int}}=\delta \Phi+H_{e c}+H_{e p h}+H_{e e}+H_{p h c}+H_{p h p h}
$$

$\delta \Phi$ is the adjustment of the core-core Coulomb interplay caused by the addition to the system's disordered atoms; it is the distinction betweenthe initial core-core repugnance Hamiltonian and the new Hamiltonian. The electronic Hamiltonian is altered by the term

$$
H_{e c}=\sum_{\substack{n i \gamma \\ n^{\prime} i^{\prime} \gamma^{\prime}}} w_{n i \gamma, n^{\prime} i^{\prime} \gamma^{\prime}} a_{n i \gamma}^{+} a_{n^{\prime} i^{\prime} \gamma^{\prime}}
$$

This term is the difference betweenthe new hopping Hamiltonian as well as the original periodic Hamiltonian. The electron-phonon interaction is described by the following equation:

$$
H_{e p h}=\sum_{\substack{n i \gamma \\ n^{\prime} i^{\prime} \gamma^{\prime}}} v_{n i \gamma, n^{\prime} i^{\prime} \gamma^{\prime}} a_{n i \gamma}^{+} a_{n^{\prime} i^{\prime} \gamma^{\prime}}
$$


A more detailed description is underneath. The Coulomb interaction Hamiltonian among electrons is described by the following expression:

$$
\begin{aligned}
H_{e e} & =\frac{1}{2} \sum_{\substack{n_{1}, n_{2}, n_{3}, n_{4}}} v_{n_{3}, n_{4}}^{(2) n_{1}, n_{2}} a_{n_{1}}^{+} a_{n_{2}}^{+} a_{n_{3}} a_{n_{4}}, \\
n=(n i \gamma) . &
\end{aligned}
$$

The adjustment of the interplay of the phonons with thecores generated by the disordering of the atoms is described by the following expression:

$$
\begin{aligned}
& H_{p h c}=\frac{1}{2} \sum_{n i \alpha} \Delta M_{n i \alpha, n^{\prime} i^{\prime} \alpha^{\prime}}^{-1} P_{n i \alpha} P_{n^{\prime} i^{\prime} \alpha^{\prime}}+ \\
& n^{\prime} i^{\prime} \alpha^{\prime} \\
& +\frac{1}{2} \sum_{\substack{n i \alpha \\
n^{\prime} i^{\prime} \alpha^{\prime}}} \Delta \Phi_{n i \alpha, n^{\prime} i^{\prime} \alpha^{\prime}} u_{n i \alpha} u_{n^{\prime} i^{\prime} \alpha^{\prime}},
\end{aligned}
$$

where

$$
\Delta M_{n i \alpha, n^{\prime} i^{\prime} \alpha^{\prime}}^{-1}=\left(\frac{1}{M_{n i^{\prime}}}-\frac{1}{M_{i}}\right) \delta_{n n^{\prime}} \delta_{i i^{\prime}} \delta_{\alpha \alpha^{\prime}}
$$

$\Delta \Phi_{n i \alpha, n^{\prime} i^{\prime} \alpha^{\prime}}=\Phi_{n i \alpha, n^{\prime} i^{\prime} \alpha^{\prime}}-\Phi_{n i \alpha, n^{\prime} i^{\prime} \alpha^{\prime}}^{(0)}$ and $M_{n i}, M_{i}$ are the atomic masses at the site (ni) for disordered alloys as well as for the ordered alloys.

The cubic anharmonic potential expressions are also included for the phonons. We assume that phonons stand small and they can be handled perturbatively through

$$
\begin{gathered}
H_{p h p h}=\frac{1}{3 !} \sum_{n i \alpha} \Phi_{n i \alpha, n^{\prime} i^{\prime} \alpha^{\prime}, n^{\prime \prime} i^{\prime \prime} \alpha^{\prime \prime}}^{(0)} u_{n i \alpha} \times \\
n^{\prime} i^{\prime} \alpha^{\prime} \\
n^{\prime \prime} i^{\prime \prime} \alpha^{\prime \prime} \\
\times u_{n^{\prime} i^{\prime} \alpha^{\prime}} u_{n^{\prime \prime} i^{\prime \prime} \alpha^{\prime \prime}}
\end{gathered}
$$

The operators $a_{n i \gamma}^{+}, a_{n i \gamma}$ produce and dismantle electrons in the state described by Vane's function.

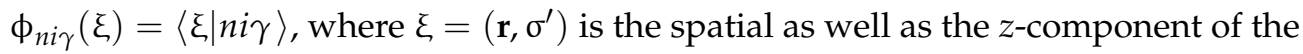
wave function of the spin coordinates. To build up the Wannier functions, we employ analytical interpretations for the wave functions of an electron in the field of atomic nuclei of kind $\lambda$, restricted in the lattice sites $(n i)$ for an ideally arranged crystal:

$$
\begin{aligned}
& \Psi_{n i \delta}\left(\mathbf{r}-\mathbf{r}_{n i}\right)=R_{\widetilde{\varepsilon} l}\left(\left|\mathbf{r}-\mathbf{r}_{n i}\right|\right) Y_{l m}\left(\mathbf{r}-\hat{-} \mathbf{r}_{n i}\right), \\
& Y_{l m}\left(\mathbf{r}-\mathbf{r}_{n i}\right)=Y_{l m}(\theta, \varphi),
\end{aligned}
$$

where $\theta, \varphi$ are the spherical angular coordinates for the vector $\mathbf{r}-\mathbf{r}_{n i}$.

Here, $\delta=\widetilde{\varepsilon} l m$ is a superindex that includes the quantum numbers for the basis energy eigenvalue $\widetilde{\varepsilon}$, the normal angular momentum quantum numbers $l$ and $m, \mathbf{r}$ is the electron location vector, and $\mathbf{r}_{n i}$ is the location vector for the atom at place $(n i)$ in balance.

$$
\begin{aligned}
& \mathbf{r}_{n i}=\mathbf{r}_{n}+\boldsymbol{\rho}_{i}, \\
& \mathbf{r}_{n}=\sum_{v} l_{v} \mathbf{a}_{v},
\end{aligned}
$$

$\mathbf{r}_{n}$ is the location vector of the junction $\mathrm{n}$ of the crystal lattice, $\boldsymbol{\rho}_{i}$ is the vector of the respective location of the junction of the sublattice $i$ at the unit cell $n$. Vectors $\boldsymbol{\rho}_{i}$ are labelled as fundamental relocation vectors of the crystal lattice, the character of which is decided by the dimension of the crystal. The coordinates $l_{v}$ of the radius vector $\mathbf{r}_{n}$ of the crystal 
lattice junction are integers. The number $v$ takes on values $v=1,2,3$ for three-dimensional crystals, $v=1,2$ for two-dimensional crystals, and $v=1$ for one-dimensional crystals. The basis orthogonalisation is carried out by means of the Lowdin approach [27]:

$$
\left|\widetilde{\psi}_{n i \delta}\right\rangle=S^{-1 / 2}\left|\psi_{n i \delta}\right\rangle, S_{n i \delta, n^{\prime} i^{\prime} \delta^{\prime}}=\left\langle\psi_{n i \delta} \mid \psi_{n / i / \delta \prime}\right\rangle
$$

where $S_{n i \delta, n^{\prime} i^{\prime} \delta^{\prime}}$ is the overlap of the integral matrix. Vanefunctions $\phi_{n i \gamma}\left(\mathbf{r}, \sigma^{\prime}\right)$, where the system's Hamiltonian is described by Equation (1), are obtained from the following equation:

$$
\phi_{n i \gamma}\left(\mathbf{r}, \sigma^{\prime}\right)=\widetilde{\psi}_{n i \delta}\left(\mathbf{r}-\mathbf{r}_{n i}\right) \chi_{\sigma}\left(\sigma^{\prime}\right)
$$

where $\chi_{\sigma}\left(\sigma^{\prime}\right)$-are the spin part of the wave function, $\gamma=\delta \sigma$.

The orthogonalised wave function may be written as follows:

$$
\widetilde{\psi}_{n_{1} i_{1} \delta_{1}}\left(r_{1}, \theta_{1}, \varphi_{1}\right)=\sum_{n_{2}, i_{2} \delta_{2}} S_{n_{2} i_{2} \delta_{2}, n_{1} i_{1} \delta_{1}}^{-\frac{1}{2}} R_{\widetilde{\varepsilon}_{2} l_{2}}\left(r_{2}\right) Y_{l_{2} m_{2}}\left(\theta_{2}, \varphi_{2}\right) .
$$

In Expression (16):

$$
\begin{gathered}
\mathbf{r}_{1}=\mathbf{r}-\mathbf{r}_{n_{1} i_{1}}, \mathbf{r}_{2}=\mathbf{r}-\mathbf{r}_{n_{2} i_{2}}=\mathbf{r}_{1}-\mathbf{r}_{n_{2} i_{2} n_{1} i_{1}}, \\
r_{2}=\left(\left(x^{1}-x_{n_{2} i_{2} n_{1} i_{1}}^{1}\right)^{2}+\left(x^{2}-x_{n_{2} i_{2} n_{1} i_{1}}^{2}\right)^{2}+\left(x^{3}-x_{n_{2} i_{2} n_{1} i_{1}}^{3}\right)^{2}\right)^{\frac{1}{2}}, \\
x^{1}=r_{1} \sin \theta_{1} \cos \varphi_{1}, x^{2}=r_{1} \sin \theta_{1} \sin \varphi_{1}, x^{3}=r_{1} \cos \theta_{1}, \\
x_{n_{2} i_{2} n_{1} i_{1}}^{\alpha}=\sum_{v}\left(l_{v}^{(2)}-l_{v}^{(1)}\right) a_{v}^{\alpha}+\rho_{i_{2}}^{\alpha}-\rho_{i_{1}}^{\alpha} \\
\cos \theta_{2}=\frac{r_{1} \cos \theta_{1}-x_{n_{2} i_{2} n_{1} i_{1}}^{3}}{r_{2}}, \\
\varphi_{2}=\arccos \frac{r_{1} \sin \theta_{1} \cos \varphi_{1}-x_{n_{2} i_{2} n_{1} i_{1}}^{1}}{r_{2}\left(1-\cos ^{2} \theta_{2}\right)^{\frac{1}{2}}} .
\end{gathered}
$$

Summation over $n_{2} i_{2}$ in Expression (16) means summation over $\mathbf{r}_{n_{2} i_{2}}$, in accordance with Formula (13).

The overlap matrix $S_{n i \delta, n^{\prime} i^{\prime} \delta^{\prime}}$ is defined by the following equation:

$$
\begin{aligned}
& S_{n_{1} i_{1} \delta_{1}, n_{2} i_{2} \delta_{2}}= \\
& \iiint R_{\widetilde{\varepsilon}_{1} l_{1}}\left(r_{1}\right) Y_{l_{1} m_{1}}^{*}\left(\theta_{1}, \varphi_{1}\right) R_{\widetilde{\varepsilon}_{2} l_{2}}\left(r_{2}\right) Y_{l_{2} m_{2}}\left(\theta_{2}, \varphi_{2}\right) r_{1}^{2} \sin \theta_{1} d r_{1} d \theta_{1} d \varphi_{1}
\end{aligned}
$$

where $r_{2}, \theta_{2}, \varphi_{2}$ are expressed through $r_{1}, \theta_{1}, \varphi_{1}$ in accordance with Formulas (17)-(19).

The procedure for calculating the matrix elements of the Hamiltonian (1), based on the use of Formulas (17)-(19), is described in Refs. [28,29].

\section{The Electron and Phonon Green's Functions}

We use Green's function formalism for our numerical computations. Ultimately, we should have the real-time retarded $G_{r}^{A B}\left(t, t^{\prime}\right)$ as well as advanced $G_{a}^{A B}\left(t, t^{\prime}\right)$ Green's functions. These are defined by the following equations [25]:

$$
\begin{aligned}
& G_{r}^{A B}\left(t, t^{\prime}\right)=-\frac{i}{\hbar} \theta\left(t-t^{\prime}\right)<\left[\widetilde{A}(t), \widetilde{B}\left(t^{\prime}\right)\right]> \\
& G_{a}^{A B}\left(t, t^{\prime}\right)=\frac{i}{\hbar} \theta\left(t^{\prime}-t\right)<[\widetilde{A}(t), \widetilde{B}(t \prime)]>.
\end{aligned}
$$

The operators are written using the Heisenberg representation:

$$
\widetilde{A}(t)=e^{i \mathrm{H} t / \hbar} A e^{-i \mathrm{H} t / \hbar}
$$


where $\hbar$ is Planck's constant, $\mathrm{H}=H-\mu_{\mathrm{e}} N_{e}, \mu_{\mathrm{e}}$ is the chemical potential of the electronic subsystem, and $N_{e}$ is the electron number operator given by

$$
N_{e}=\sum_{n i \gamma} a_{n i \gamma}^{+} a_{n i \gamma}
$$

It is well-known that a commutator or anticommutator is described through

$$
[A, B]=A B \mp B A
$$

In this expression, a commutator is employed for the Bose operators $(-)$, whereas the anticommutator is employed for Fermi operators $(+)$. Here, a symbol $\theta(t)$ is Heaviside's unit step function. Here, the angle brackets $\langle\ldots\rangle$ describe the thermal averaging connectedto a density matrix $\rho$

$$
<A>=\operatorname{Tr}(\rho A), \rho=e^{(\Omega-\mathrm{H}) / \Theta}
$$

Here, $\Omega$ is a thermodynamic potential for the system described using $\exp (\Omega / \Theta)=\operatorname{Trexp}(-\mathrm{H} / \Theta)$ and $\Theta=k_{b} T$, with $k_{b}$ as the Boltzmann's constant, but $T$ defining a temperature. It is worth noting that although real-time Green's functions areseemingly dependent on the two different time moments, due to time-translational invariance for the equilibrium systems, in reality, this function is only dependent onthe time difference $t-t^{\prime}$.

The approach developed by us forcomputing the real-time Green's functions acts in accordance with the standard one. Namely, as a starting point, we determined the thermal Green's functions (defined below) and analytically developed them to the real-time by means of the well-knownspectral relations. The thermal Green's function is written as follows:

$$
G^{A B}(\tau, \tau \prime)=-<T_{\tau} \widetilde{A}(\tau) \widetilde{B}(\tau \prime)>
$$

In this equation, the imaginary-time operator $\widetilde{A}(\tau)$ is obtained from a real-time Heisenberg description using the following substitution $t=-i \hbar \tau$. Consequently,

$$
\widetilde{A}(\tau)=e^{\mathrm{H} \tau} A e^{-\mathrm{H} \tau}
$$

Furthermore, the time-ordering operator satisfies the following relation:

$$
\begin{aligned}
& T_{\tau} \widetilde{A}(\tau) \widetilde{B}(\tau \prime)=\theta(\tau-\tau \prime) \widetilde{A}(\tau) \widetilde{B}(\tau \prime)+ \\
& \pm \theta(\tau \prime-\tau) \widetilde{B}(\tau \prime) \widetilde{A}(\tau)
\end{aligned}
$$

In this equation, the plus sign is used for the Bose operators, whereas the minus sign is used for the Fermi operators. As a next step, we introduced the operator in the interaction representation

$$
\sigma(\tau)=e^{\mathrm{H}_{0} \tau} e^{-\mathrm{H} \tau}
$$

with $\mathrm{H}=\mathrm{H}_{0}+H_{\text {int }}$ and $\mathrm{H}_{0}=H_{0}-\mu_{e} N_{e}$. Applying the differentiation for $\sigma(\tau)$ in Equation (29) regarding the $\tau$ and after integrating from 0 , and taking into account the boundary condition $\sigma(0)=1$, we obtain

$$
\sigma(\tau)=T_{\tau} \exp \left[-\int_{0}^{\tau} H_{\text {int }}(\tau \prime) d \tau \prime\right]
$$

where $H_{\text {int }}(\tau)=e^{\mathrm{H}_{0} \tau} H_{\text {int }} e^{-\mathrm{H}_{0} \tau}$. Employing this result yields

$$
\widetilde{A}(\tau)=\sigma^{-1}(\tau) A(\tau) \sigma(\tau)
$$


where $A(\tau)$ is Heisenberg presentation regarding the non-interacting Hamiltonian. Replacing the obtained results in the descriptionof the thermal Green's functiongeneratesanother interaction-presentation form for the Green's function, specified as follows:

$$
G^{A B}(\tau, \tau \prime)=-\frac{<T_{\tau} A(\tau) B(\tau \prime) \sigma(1 / \Theta)>_{0}}{<\sigma(1 / \Theta)>_{0}}
$$

In this equation, time dependence regarding the non-interacting Hamiltonian, as well as the trace over all states regarding the non-interacting states, is as follows:

$$
<A>_{0}=\operatorname{Tr}\left(\rho_{0} A\right), \rho_{0}=e^{\left(\Omega_{0}-\mathrm{H}_{0}\right) / \Theta}
$$

The previous equation creates an opening point for the perturbative extension used by us. This diagrammethod is created by expanding $\sigma(\tau)$ in a power series using the terms of $H_{\text {int }}(\tau)$ and then applying Wick's theorem in order to estimate the resulting operator averages $[25,26]$.

Summing up the indicated series, by using the standard relation between the spectral representations of the temperature and real-time Green's functions, and performing an analytical continuation on the real axis, we obtain the following equations for the retarded Green's functions [25] (it is worth noting that the dependence from $r$ is subdued):

$$
\begin{gathered}
G^{a a^{+}}(\varepsilon)=\left[\varepsilon-H_{0}^{(1)}-\left(w+\Sigma_{e p h}(\varepsilon)+\Sigma_{e e}(\varepsilon)\right)\right]^{-1} \\
G^{u u}(\varepsilon)=\left[\omega^{2} M^{(0)}-\Phi^{(0)}-\left(\frac{\varepsilon^{2}}{\hbar^{2}} \Delta M+\Delta \Phi+\Sigma_{p h e}(\varepsilon)+\Sigma_{p h p h}(\varepsilon)\right)\right]^{-1} \\
G^{P P}(\varepsilon)=\frac{\varepsilon^{2}}{\hbar^{2}}\left(M^{(0)}\right)^{2} G^{u u}(\varepsilon),
\end{gathered}
$$

where

$$
\begin{aligned}
& H_{0}^{(1)}=\left\|h_{n i \gamma, n^{\prime} i^{\prime} \gamma^{\prime}}^{(0)}\right\| \\
& \Phi^{(0)}=\left\|\Phi^{(0)}{ }_{n i \alpha, n / i \prime \alpha)}\right\| \\
& M^{(0)}=\left\|M_{i} \delta_{n n^{\prime}} \delta_{i i \prime} \delta_{\alpha \alpha \prime}\right\| \\
& \Delta M=\left\|\left(M_{i}-M_{n i}\right) \delta_{n n \prime} \delta_{i i \prime} \delta_{\alpha \alpha}\right\|, \varepsilon=\hbar \omega .
\end{aligned}
$$

Here, $G^{a a^{+}}(\varepsilon), G^{u u}(\varepsilon)$, and $G^{P P}(\varepsilon)$ are the real-frequency descriptions for the singleparticle Green's function ofelectrons, the coordinate-coordinate and momentum-momentum, as well as the Green's functions for the phonons. $\Sigma_{e p h}(\varepsilon), \Sigma_{p h e}(\varepsilon), \Sigma_{e e}(\varepsilon)$, and $\Sigma_{p h p h}(\varepsilon)$ are the corresponding self-energies of the electron-phonon, phonon-electron, electron-electron, as well as phonon-phonon interactions. The electron Green's functions are infinite matrices. They have the indices specified by the lattice site $n$ and the basis site $i$ as well as the other quantum number $\gamma$. Likewise, the phonon Green's functions are also boundless matrices with the matching lattice as well as basis site dependence, plus a dependence on the spatial coordinate direction $\alpha$. The mass operator of Green's function of electrons for the electron-phonon interplay $\Sigma_{e p h}(\tau, \tau /)$ is characterised by the diagram in Refs. [28,29]. Straightforwardly, the electron-phonon self-energy becomes

$$
\begin{aligned}
& \Sigma_{\mathrm{e} p h n i \gamma, n / i \gamma \gamma}(\varepsilon)=-\frac{1}{4 \pi i} \int_{-\infty}^{\infty} d \varepsilon / \operatorname{coth}\left(\frac{\varepsilon \prime}{2 \Theta}\right) \Gamma_{n i \gamma_{1}, n_{3} i_{3} \gamma_{3}}^{(0) n_{1} i_{\alpha} \alpha_{1}} \times \\
& \times\left[G_{n_{1} i_{1} \alpha_{1}, n_{2} i_{2} \alpha_{2}}^{u u}(\varepsilon \prime)-G_{n_{1} i_{1} \alpha_{1}, n_{2} i_{2} \alpha_{2}}^{u u}(\varepsilon \prime)\right] G_{n_{3} i_{3} \gamma_{3}, n_{4} i_{4} \gamma_{4}}^{a a^{+}} \times
\end{aligned}
$$

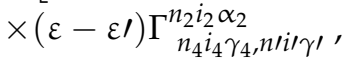

$$
\begin{aligned}
& \Gamma_{n i \gamma_{1}, n_{3} i_{3} \gamma_{3}}^{(0) n_{1} i_{1} \alpha_{1}}=\boldsymbol{v}_{n i \gamma, n_{3} i_{3} \gamma_{3}}^{\prime n_{1} i_{1} \alpha_{1}} .
\end{aligned}
$$


There, we added repeated indices. Summation is conducted over repeated indices. Phonon-electron interplay is reported using the diagram in Refs. [28,29].

The mass operator that characterises the electron-electron interplay is described as follows:

$$
\begin{aligned}
& \Sigma_{\text {phe } n i \alpha, n / i \prime \alpha \prime}(\varepsilon)=\frac{1}{2 \pi i} \int_{-\infty}^{\infty} d \varepsilon^{\prime} f(\varepsilon \prime) \Gamma_{n_{2} i_{2} \gamma_{2}, n_{1} i_{1} \gamma_{1}}^{(0) n i \alpha} \times \\
& \times\left\{\left[G_{n_{1} i_{1} \gamma_{1}, n_{3} i_{3} \gamma_{3}}^{a{ }^{+}}(\varepsilon+\varepsilon \prime)-G_{n_{1} i_{1} \gamma_{1}, n_{3} i_{3} \gamma_{3}}^{a{ }^{+} *}(\varepsilon+\varepsilon \prime)\right] \times\right. \\
& \times G_{n_{4} i_{4} \gamma_{4}, n_{2} i_{2} \gamma_{2}}^{a{ }^{+} *}(\varepsilon \prime)+G_{n_{1} i_{1} \gamma_{1}, n_{3} i_{3} \gamma_{3}}^{a a^{+}}(\varepsilon+\varepsilon \prime) \times \\
& \left.\times\left[G_{n_{4} i_{4} \gamma_{4}, n_{2} i_{2} \gamma_{2}}^{a a}(\varepsilon \prime)-G_{n_{4} i_{4} \gamma_{4}, n_{2} i_{2} \gamma_{2}}^{a a^{+} *}(\varepsilon \prime)\right]\right\} \times \\
& \times \Gamma_{n_{3} i_{3} \gamma_{3}, n_{4} i_{4} \gamma_{4}}^{n / i \gamma_{4}} .
\end{aligned}
$$

where $f(\varepsilon)$ is the world-well-known Fermi Dirac distribution function.

Diagrams of the mass operator $\Sigma_{e e}(\tau, \tau /)$ that characterise the electron-electroninterplay are depicted in Refs. [28,29].

The mass operator that characterises the electron-electron interplay is described as follows:

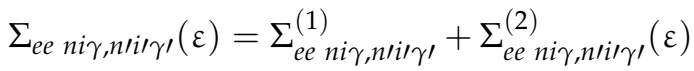

$$
\begin{aligned}
& \Sigma_{\text {ee } n, n \prime}^{(1)}=-\frac{1}{2 \pi i} \int_{-\infty}^{\infty} d \varepsilon \prime f(\varepsilon \prime) \Gamma_{n, n \prime}^{(0) n_{2}, n_{1}}\left[G_{n_{1}, n_{2}}^{a a^{+}}\left(\varepsilon^{\prime}\right)-G_{n_{1}, n_{2}}^{a a^{+}}(\varepsilon \prime)\right] \\
& \Sigma_{\mathrm{ee}}^{(2)} n_{n \prime}(\varepsilon)=\left(\frac{1}{2 \pi i}\right)^{2} \int_{-\infty}^{\infty} d \varepsilon_{1} \int_{-\infty}^{\infty} d \varepsilon_{2} f\left(\varepsilon_{1}\right) f\left(\varepsilon_{2}\right) \Gamma_{n_{2}, n_{1}}^{(0)} n_{n} \\
& \times\left[G_{n_{2}, n_{5}}^{a a^{+}}\left(\varepsilon-\varepsilon_{1}-\varepsilon_{2}\right) G_{n_{1}, n_{4}}^{a a^{+} *}\left(\varepsilon_{1}\right)-\right. \\
& \left.-G_{n_{2}, n_{5}}^{a a^{+} *}\left(\varepsilon-\varepsilon_{1}-\varepsilon_{2}\right) G_{n_{1}, n_{4}}^{a a^{+}}\left(\varepsilon_{1}\right)\right] \times \\
& \times\left[G_{n_{6}, n_{3}}^{a a^{+}}\left(\varepsilon_{2}\right)-G_{n_{6}, n_{3}}^{a a^{+}}\left(\varepsilon_{2}\right)\right] \\
& \text { - } \left.G_{n_{2}, n_{5}}^{a a^{+}}\left(\varepsilon-\varepsilon_{1}-\varepsilon_{2}\right)-G_{n_{2}, n_{5}}^{a a^{+}}\left(\varepsilon-\varepsilon_{1}-\varepsilon_{2}\right)\right] \times \\
& \times\left[G_{n_{1}, n_{4}}^{a a^{+}}\left(\varepsilon_{1}\right) G_{n_{6}, n_{3}}^{a a^{+}}\left(\varepsilon_{2}\right)\right. \\
& \left.\left.-G_{n_{1}, n_{4}}^{a a^{+}}{ }^{*}\left(\varepsilon_{1}\right) G_{n_{6}, n_{3}}^{a a^{+}}\left(\varepsilon_{2}\right)\right]\right\} \Gamma^{n_{5}, n_{6}}{ }_{n_{4}, n^{\prime \prime}} \\
& \Gamma_{n_{2}, n_{1}}^{(0) n, n_{3}}=\widetilde{v}_{n_{2}, n_{1}}^{(2) n, n_{3}}=v_{n_{2}, n_{1}}^{(2) n, n_{3}}-v_{n_{1}, n_{2}}^{(2) n, n_{3}} n=n i \gamma
\end{aligned}
$$

Related outcomes to the addition for the phonon self-energy $\Sigma_{\text {phph }}(\varepsilon)$, coming out of the phonon-phonon coupling, arealso described in Ref. [25].

We now obtain equations for the vertex parts of the mass operators of electron-phonon, phonon-electron, and electron-electron interactions, represented by Equations (41), (43), and (46). In general, the renormalisation for the apex of the functions entering Equations (41), (43), and (46) for the mass operators could be carried out employing the diagrams in Refs. [28,29]. Namely, we have

$$
\begin{aligned}
& \Gamma_{n_{3} i_{3} \gamma_{3}, n_{4} i_{4} \gamma_{4}}^{n^{\prime} \gamma^{\prime}}=\Gamma_{n_{3} i_{3} \gamma_{3}, n_{4} i_{4} \gamma_{4}}^{(0) n^{\prime} i^{\prime}}-\frac{1}{2 \pi i} \int_{-\infty}^{\infty} d \varepsilon f(\varepsilon) \Gamma_{n_{5} i_{5} \gamma_{5}, n_{6} i_{6} \gamma_{6}}^{(0) n^{\prime}{ }^{\prime} \alpha^{\prime}} \\
& \times\left[G_{n_{6} i_{6} \gamma_{6}, n_{7} i_{7} \gamma_{7}}^{a{ }^{+}}(\varepsilon) G_{n_{8} i_{8} \gamma_{8}, n_{5} i_{5} \gamma_{5}}^{a a^{+}}(\varepsilon)-G_{n_{6} i_{6} \gamma_{6}, n_{7} i_{7} \gamma_{7}}^{a a+}(\varepsilon) G_{n_{8} i_{8} \gamma_{8}, n_{5} i_{5} \gamma_{5}}^{a a^{+} *}(\varepsilon)\right]
\end{aligned}
$$

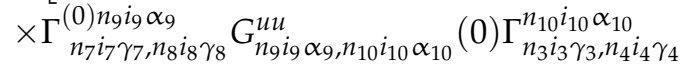


and

$$
\begin{aligned}
& \Gamma_{n_{4}, n^{\prime}}^{n_{5}, n_{6}}=\Gamma_{n_{4}, n^{\prime}}^{(0) n_{5}, n_{6}}-\frac{1}{2 \pi i} \int_{-\infty}^{\infty} d \varepsilon f(\varepsilon) \Gamma_{n_{4}, n_{8}}^{(0) n_{5}, n_{7}} \\
& \times\left[G_{n_{7}, n_{9}}^{a a^{+}}(\varepsilon) G_{n_{8}, n_{10}}^{a a^{+} *}(\varepsilon)-G_{n_{7}, n_{9}}^{a a^{+} *}(\varepsilon) G_{n_{8}, n_{10}}^{a a^{+}}(\varepsilon)\right] \\
& \times \Gamma_{n_{10}, n_{6}, n}^{n_{1}, n=n i \gamma .}
\end{aligned}
$$

Summation is implied over repeated indices in Expressions (48) and (49).

The Fermi level $\varepsilon_{F} \equiv \mu_{\mathrm{e}}$ for the arrangement is resolved using the following equation:

$$
\begin{gathered}
<Z>=\int_{-\infty}^{\infty} f(\varepsilon) g_{e}(\varepsilon) d \varepsilon \\
f(\varepsilon)=\frac{1}{\exp \left(\frac{\varepsilon-\varepsilon_{F}}{\Theta}\right)+1}
\end{gathered}
$$

where $<Z>$ means the mean number of electrons per atom and $g_{e}(\varepsilon)$ is the many-body electronic density of states, which fulfil the relation

$$
g_{e}(\varepsilon)=-\frac{1}{\pi v N} \operatorname{Im} \operatorname{Tr}\left\langle G^{a a^{+}}(\varepsilon)\right\rangle_{c}
$$

where $\langle\ldots\rangle_{c}$ indicates configurational calibrating of the disorder, $N$ is the amount of the primitive lattice cells, and $v$ is the number of atoms by the primitive cell. We let the letter $c$ fall on the configurational calibrating in order to make our equations more simple. In Equation (50), variable $\langle Z\rangle$ describes the average amount ofelectrons on the atom.

\section{Density of Electronic and Phononic States}

In Equations (34) and (35), due to the introduction of the mass operator equal to the sum of the one-site operators as well as by selecting as a zero approximation the effective medium of Green's function, the cluster expansion for Green's functions $G^{a a^{+}}(\varepsilon)$ and $G^{u u}(\varepsilon)$ was performed. The described expansion is the cluster expansion's generalisation for Green's function $G^{a a^{+}}(\varepsilon)$ of the single-particle Hamiltonian. Green's functions of the effective environment are defined by the expressions:

$$
\begin{aligned}
\widetilde{G}^{a a^{+}}(\varepsilon) & =\left[\varepsilon-h^{(0)}-\widetilde{\Sigma}_{e p h}(\varepsilon)-\widetilde{\Sigma}_{e e}(\varepsilon)-\sigma_{e}(\varepsilon)\right]^{-1} \\
\widetilde{G}^{u u}(\varepsilon) & =\left[\frac{\varepsilon^{2}}{\hbar^{2}} M^{(0)}-\Phi^{(0)}-\widetilde{\Sigma}_{p h e}(\varepsilon)-\sigma_{p h}(\varepsilon)\right]^{-1}
\end{aligned}
$$

Expressions for the operators $\widetilde{\Sigma}_{e p h}(\varepsilon), \widetilde{\Sigma}_{p h e}(\varepsilon)$, and $\widetilde{\Sigma}_{e e}(\varepsilon)$ are obtained from the expressions for the mass operators $\Sigma_{e p h}(\varepsilon), \Sigma_{\text {phe }}(\varepsilon)$, and $\Sigma_{e e}(\varepsilon)(41)-(47)$ by replacing the vertex parts $\Gamma_{n i \gamma, n_{3} i_{3} \gamma_{3}}^{(0) n_{1} i_{1} \alpha_{1}}, \Gamma_{n_{2}, n_{1}}^{(0) n, n_{3}}, n \equiv n i \gamma$ by their expressions for ideally ordered crystals and replacing the Green's functions $G^{a a^{+}}(\varepsilon)$ and $G^{u u}(\varepsilon)$ with the Green's functions of the effective medium, $\widetilde{G}^{a a^{+}}(\varepsilon)$ and $\widetilde{G}^{u u}(\varepsilon)$. Expressions for operators $\sigma_{e}(\varepsilon)$ and $\sigma_{p h}(\varepsilon)$ in Formulas (53) and (54) will be given below.

The Green's functions in Equations (34) and (35) persuade the Dyson equation, which may be written in terms of the T-matrix through

$$
G(\varepsilon)=\widetilde{G}(\varepsilon)+\widetilde{G}(\varepsilon) T(\varepsilon) \widetilde{G}(\varepsilon)
$$

in this expression, the $T$-matrix is characterised by a series, where each termexpresses the scattering of clusters with different numbers of nodes schematically described as follows:

$$
T=\sum_{\left(n_{1} i_{1}\right)} t^{n_{1} i_{1}}+\sum_{\left(n_{1} i_{1}\right) \neq\left(n_{2} i_{2}\right)} T^{(2) n_{1} i_{1}, n_{2} i_{2}}+\ldots
$$


Here, we have

$$
T^{(2) n_{1} i_{1}, n_{2} i_{2}}=\left[I-t^{n_{1} i_{1}} \widetilde{G} t^{n_{2} i_{2}} \widetilde{G}\right]^{-1} t^{n_{1} i_{1}} \widetilde{G} t^{n_{2} i_{2}}\left[I+\widetilde{G} t^{n_{1} i_{1}}\right]
$$

where $t^{n_{1} i_{1}}$ is the on-site scattering operator, which is described as follows:

$$
t^{n_{1} i_{1}}=\left[I-\left(\Sigma^{n_{1} i_{1}}-\sigma^{n_{1} i_{1}}\right) \widetilde{G}\right]^{-1}\left(\Sigma^{n_{1} i_{1}}-\sigma^{n_{1} i_{1}}\right)
$$

The self-energy employed in Equation (34), $\Sigma_{e}^{n_{1} i_{1}}(\varepsilon)$, satisfies

$$
w+\Sigma_{e p h}(\varepsilon)+\Sigma_{e e}(\varepsilon)-\widetilde{\Sigma}_{e p h}(\varepsilon)-\widetilde{\Sigma}_{e e}(\varepsilon)=\sum_{\left(n_{1} i_{1}\right)} \Sigma_{e}^{n_{1} i_{1}}(\varepsilon)
$$

For the electrons. For the phonons, we have

$$
\begin{aligned}
& \frac{\varepsilon^{2}}{\hbar^{2}} \Delta M+\Delta \Phi+\Sigma_{p h e}(\varepsilon)+\Sigma_{p h p h}(\varepsilon)- \\
& -\widetilde{\Sigma}_{p h e}(\varepsilon)-\widetilde{\Sigma}_{p h p h}(\varepsilon)=\sum_{\left(n_{1} i_{1}\right)} \sum_{p h}^{n_{1} i_{1}}(\varepsilon)
\end{aligned}
$$

Using Equations (34) and (59),we obtain the expression for the intrinsic energy part $\sum_{\text {eni } \gamma_{, n i} \lambda^{\prime} \gamma^{\prime}}^{\lambda n_{1} i_{1}}(\varepsilon)$, which describes the scattering of electrons:

$$
\begin{aligned}
& \sum_{e n_{1} i_{1} \gamma_{1}, n_{2} i_{2} \gamma_{2}}^{\lambda m_{\lambda} n i}(\varepsilon)=w_{n_{1} i_{1} \gamma_{1}, n_{2} i_{2} \gamma_{2}}^{\lambda n i}+
\end{aligned}
$$

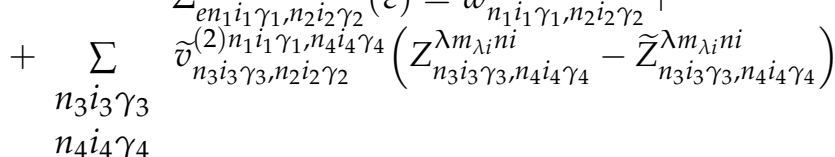

where

$$
Z_{n_{3} i_{3} \gamma_{3}, n_{4} i_{4} \gamma_{4}}^{\lambda m_{\lambda} i n}=-\left.\frac{1}{\pi} \int_{-\infty}^{\infty} f\left(\varepsilon, \varepsilon_{F}\right) \operatorname{Im}\left\langle G_{n_{3} i_{3} \gamma_{3}, n_{4} i_{4} \gamma_{4}}^{a a^{+}}(\varepsilon)\right\rangle\right|_{(n i) \in \lambda m_{\lambda \mathrm{i}}} d \varepsilon
$$

The value of $\widetilde{Z}_{n_{3} i_{3} \gamma_{3}, n_{4} i_{4} \gamma_{4}}^{\lambda \lambda_{1} i n}$ in Equation (61) is obtained from Equation (62) via replacing of the fullGreen's function via the effective medium Green's function. The matrix diagonal elements $Z_{n_{3} i_{3} \gamma_{3}, n_{4} i_{4} \gamma_{4}}^{\lambda m_{\lambda} n i}$ in Equation (62) are similar to the occupation numbers for the electron states $Z_{n i \delta \sigma}^{\lambda m_{\lambda i}}$ (see Refs. [28,29]).

Using Equations (35) and (60),we obtain the expression for the intrinsic energy part $\sum_{\text {phni } \lambda_{, n / i / \alpha}}^{\lambda n_{1} i_{1}}(\varepsilon)$, which describes the scattering of phonons:

$$
\Sigma_{p h n i \alpha, n / i^{\prime} \alpha \prime}^{\lambda n_{1} i_{1}}(\varepsilon)=\frac{\varepsilon^{2}}{\hbar^{2}}\left(M_{i_{1}}-M_{\lambda}\right) \delta_{n n \prime} \delta_{i i \prime} \delta_{\alpha \alpha \prime}
$$

It should be noted that, in the infinite crystal limit, on the right-hand side of Equations (61) and (63), the terms inversely proportional to the number of lattice sites are neglected. We require the fulfilment of the condition

$$
\left\langle t^{0 i_{1}}\right\rangle=0
$$

from which follows the system of coupled equations for the operator in Formulas (53) and (54)

$$
\begin{aligned}
& \sigma_{e}^{0 i_{1}}(\varepsilon)=\left\langle\left[1-\left(\Sigma_{e}^{0 i_{1}}(\varepsilon)-\sigma_{e}^{0 i_{1}}(\varepsilon)\right) \widetilde{G}^{a a^{+}}(\varepsilon)\right]^{-1}\right\rangle^{-1} \times \\
& \times\left\langle\left[1-\left(\Sigma_{e}^{0 i_{1}}(\varepsilon)-\sigma_{e}^{0 i_{1}}(\varepsilon)\right) \widetilde{G}^{a a^{+}}(\varepsilon)\right]^{-1} \Sigma_{e}^{0 i_{1}}(\varepsilon)\right\rangle^{-}
\end{aligned}
$$




$$
\begin{aligned}
& \sigma_{p h}^{0 i_{1}}(\varepsilon)=\left\langle\left[1-\left(\Sigma_{p h}^{0 i_{1}}(\varepsilon)-\sigma_{p h}^{0 i_{1}}(\varepsilon)\right) \widetilde{G}^{u u}(\varepsilon)\right]^{-1}\right\rangle^{-1} \times \\
& \times\left\langle\left[1-\left(\Sigma_{p h}^{0 i_{1}}(\varepsilon)-\sigma_{p h}^{0 i_{1}}(\varepsilon)\right) \widetilde{G}^{u u}(\varepsilon)\right]^{-1} \Sigma_{p h}^{0 i_{1}}(\varepsilon)\right\rangle
\end{aligned}
$$

The matrix elements for Green's function for the electron subsystem of the effective medium can be effectively computed by means of Fourier transformation:

$$
\begin{gathered}
\widetilde{G}_{n i \gamma, n^{\prime} i^{\prime} \gamma^{\prime}}^{a a^{+}}(\varepsilon)=\frac{1}{N} \sum_{\mathbf{k}} \widetilde{G}_{i \gamma_{,} i \gamma \gamma^{\prime}}^{a a^{+}}(\mathbf{k}, \varepsilon) e^{i \mathbf{k}\left(\mathbf{r}_{n i}-\mathbf{r}_{n i i}\right)}, \\
\widetilde{G}^{a a^{+}}(\mathbf{k}, \varepsilon)=(\varepsilon-\widetilde{H}(\mathbf{k}, \varepsilon))^{-1},
\end{gathered}
$$

where

$$
\widetilde{H}(\mathbf{k}, \varepsilon)=h^{(0)}(\mathbf{k})+\widetilde{\Sigma}_{e p h}(\mathbf{k}, \varepsilon)+\widetilde{\Sigma}_{e e}(\mathbf{k}, \varepsilon)+\sigma_{e}(\mathbf{k}, \varepsilon)
$$

$N$ is the number of primitive unit cells. We performed an equal course of action for the effective medium-phonon Green's function, which satisfies

$$
\begin{gathered}
\widetilde{G}_{n i \alpha, n i^{\prime \prime \alpha \prime}}^{u u}(\varepsilon)=\frac{1}{N} \sum_{\mathbf{k}} \widetilde{G}_{i \alpha, i \prime \alpha \prime}^{u u}(\mathbf{k}, \varepsilon) e^{i \mathbf{k}\left(\mathbf{r}_{n i}-\mathbf{r}_{n i l i}\right),} \\
\widetilde{G}^{u u}(\mathbf{k}, \varepsilon)=\left(\frac{\varepsilon^{2}}{\hbar^{2}} M^{(0)}-\widetilde{\Phi}(\mathbf{k}, \varepsilon)\right)^{-1},
\end{gathered}
$$

There, we have

$$
\begin{gathered}
\widetilde{\Phi}(k, \varepsilon)=\Phi^{(0)}(\mathbf{k})+\widetilde{\Sigma}_{p h e}(\mathbf{k}, \varepsilon)+\widetilde{\Sigma}_{p h p h}(\mathbf{k}, \varepsilon)+\sigma_{p h}(\varepsilon) \\
M_{i \alpha, i^{\prime} \alpha^{\prime}}^{(0)}=M_{i} \delta_{i i^{\prime}} \delta_{\alpha \alpha^{\prime}}
\end{gathered}
$$

It is worth noticing that the wave vector $\mathbf{k}$ fluctuates inside the first Brillouin zone. The Fourier transform for a mass operator of the electron-phonon interplay has the following form:

$$
\begin{aligned}
& \widetilde{\Sigma}_{\mathrm{eph} i \gamma, i / \gamma^{\prime}}(\mathbf{k}, \varepsilon)=-\frac{1}{4 \pi i} \frac{1}{N} \int_{-\infty}^{\infty} d \varepsilon_{1} \operatorname{coth}\left(\frac{\varepsilon_{1}}{2 \Theta}\right) \\
& \times \sum_{\mathbf{k}_{1}} \Gamma_{i \gamma, i_{3} \gamma_{3}}^{(0) i_{1} \alpha_{1}}\left(-\mathbf{k}, \mathbf{k}-\mathbf{k}_{1}\right)\left[\widetilde{G}_{i_{1} \alpha_{1}, i_{2} \alpha_{2}}^{u u}\left(\mathbf{k}_{1} \varepsilon_{1}\right)-\widetilde{G}_{i_{1} \alpha_{1}, i_{2} \alpha_{2}}^{u u *}\left(\mathbf{k}_{1} \varepsilon_{1}\right)\right] \\
& \times \widetilde{G}_{i_{3} \gamma_{3}, i_{4} \gamma_{4}}^{a a^{+}}\left(\mathbf{k}-\mathbf{k}_{1}, \varepsilon-\varepsilon_{1}\right) \widetilde{\Gamma}_{i_{4} \gamma_{4}, i \gamma^{\prime}}^{i_{2} \alpha_{2}}\left(-\mathbf{k}+\mathbf{k}_{1}, \mathbf{k}\right) .
\end{aligned}
$$

The Fourier transform of the phonon-electron interaction mass operator is:

$$
\begin{aligned}
& \widetilde{\Sigma}_{\text {phe } n i \alpha, n / i \prime \alpha \prime}(\mathbf{k}, \varepsilon)=\frac{1}{2 \pi i} \frac{1}{N} \int_{-\infty}^{\infty} d \varepsilon_{1} f\left(\varepsilon_{1}\right) \sum \Gamma_{i_{2} \gamma_{2}, i_{1} \gamma_{1}}^{(0) i \alpha}\left(-\mathbf{k}_{1}, \mathbf{k}+\mathbf{k}_{1}\right) \\
& \times\left\{\left[\widetilde{G}_{i_{1} \gamma_{1}, i_{3} \gamma_{3}}^{a a^{+}}\left(\mathbf{k}+\mathbf{k}_{1}, \varepsilon+\varepsilon_{1}\right)-\widetilde{G}_{i_{1} \gamma_{1}, i_{3} \gamma_{3}}^{a a^{+}{ }^{\prime}}\left(\mathbf{k}+\mathbf{k}_{1}, \varepsilon+\varepsilon_{1}\right)\right]\right. \\
& \times \widetilde{G}_{i_{4} \gamma_{4}, i_{2} \gamma_{2}}^{a+}\left(\mathbf{k}_{1}, \varepsilon_{1}\right)+\widetilde{G}_{i_{1} \gamma_{1}, i_{3} \gamma_{3}}^{a a^{+}}\left(\mathbf{k}+\mathbf{k}_{1}, \varepsilon+\varepsilon_{1}\right) \\
& \left.\times\left[\widetilde{G}_{i_{4} \gamma_{4}, i_{2} \gamma_{2}}^{a a^{+}}\left(\mathbf{k}_{1}, \varepsilon_{1}\right)-\widetilde{G}_{i_{4} \gamma_{4}, i_{2} \gamma_{2}}^{a a^{+}}\left(\mathbf{k}_{1}, \varepsilon_{1}\right)\right]\right\} \widetilde{\Gamma}_{i_{3} \gamma_{3}, i_{4} \gamma_{4}}^{i \prime \gamma^{\prime}}\left(-\mathbf{k}-\mathbf{k}_{1}, \mathbf{k}_{1}\right) .
\end{aligned}
$$


The vertex parts of the mass operators of the electron-phonon as well as phonon interplays are determined using the following equation:

$$
\begin{aligned}
& \widetilde{\Gamma}_{i_{3} \gamma_{3}, i_{4} \gamma_{4}}^{n i^{\prime} \alpha^{\prime}}\left(\mathbf{k}_{1}, \mathbf{k}_{2}\right)=\Gamma_{i_{3} \gamma_{3}, i_{4} \gamma_{4}}^{(0) i^{\prime} \alpha^{\prime}}\left(\mathbf{k}_{1}, \mathbf{k}_{2}\right)-\frac{1}{2 \pi i} \frac{1}{N} \int_{-\infty}^{\infty} d \varepsilon f(\varepsilon) \\
& \times \sum \Gamma_{i_{5} \gamma_{5}, i_{6} \gamma_{6}}^{(0))^{\prime}{ }^{\prime}}\left(\mathbf{k}_{1}+\mathbf{k}_{2}-\mathbf{k}_{5}, \mathbf{k}_{5}\right) \\
& \times\left[\widetilde{G}_{i_{6} \gamma_{6}, i_{7} \gamma_{7}}^{a \gamma^{+}}\left(\mathbf{k}_{5}, \varepsilon\right) \widetilde{G}_{i_{8} \gamma_{8}, i_{5} \gamma_{5}}^{a a^{+}}\left(-\mathbf{k}_{1}-\mathbf{k}_{2}+\mathbf{k}_{5} \varepsilon\right)\right. \\
& \left.-\widetilde{G}_{i_{6} \gamma_{6}, i_{7} \gamma_{7}}^{a a^{+} *}\left(\mathbf{k}_{5}, \varepsilon\right) \widetilde{G}_{i_{8} \gamma_{8}, i_{5} \gamma_{5}}^{a a^{+} *}\left(-\mathbf{k}_{1}-\mathbf{k}_{2}+\mathbf{k}_{5} \varepsilon\right)\right] \\
& \times \Gamma_{i_{7} \gamma_{7}, i_{8} \gamma_{8}}^{(0) i_{9} \alpha_{9}}\left(-\mathbf{k}_{5},-\mathbf{k}_{1}-\mathbf{k}_{2}+\mathbf{k}_{5}\right) \widetilde{G}_{i_{9} \alpha_{9}, i_{10} \alpha_{10}}^{u u}\left(\mathbf{k}_{1}+\mathbf{k}_{2}, 0\right) \\
& \times \widetilde{\Gamma}_{i_{3} \gamma_{3}, i_{4} \gamma_{4}}^{i_{10} \alpha_{10}}\left(\mathbf{k}_{1}, \mathbf{k}_{1}+\mathbf{k}_{2}\right) \text {. }
\end{aligned}
$$

In Expressions (74)-(76)

$$
\begin{aligned}
& \Gamma_{i_{1} \gamma_{1}, i_{2} \gamma_{2}}^{(0) i \alpha}\left(\mathbf{k}_{1}, \mathbf{k}_{2}\right)= \\
& \sum_{n_{1}, n_{2}}^{i_{1} \gamma_{1}, i_{2} \gamma_{2}} v_{n_{1} i_{1} \gamma_{1}, n_{2} i_{2} \gamma_{2}}^{\prime \prime n} \exp \left(i \mathbf{k}_{1}\left(\mathbf{r}_{n_{1} i_{1}}-\mathbf{r}_{n i}\right)+i \mathbf{k}_{2}\left(\mathbf{r}_{n_{2} i_{2}}-\mathbf{r}_{n i}\right)\right) \text {. }
\end{aligned}
$$

The Fourier transform for the mass operator of the electron-electron interplay can be written using the following expression:

$$
\begin{aligned}
& \widetilde{\Sigma}_{e e i \gamma, i^{\prime} \gamma^{\prime}}(\mathbf{k}, \varepsilon)=\widetilde{\Sigma}_{e e i \gamma, i \gamma^{\prime}}^{(1)}(\mathbf{k})+\widetilde{\Sigma}_{e e i \gamma, i \prime \gamma^{\prime}}^{(2)}(\mathbf{k}, \varepsilon) \\
& \widetilde{\Sigma}_{\mathrm{ee} \mathrm{i} \gamma, i \prime \gamma^{\prime}}^{(1)}(\mathbf{k})=-\frac{1}{2 \pi i} \frac{1}{N} \\
& \times \int_{-\infty}^{\infty} d \varepsilon_{1} f\left(\varepsilon_{1}\right) \sum_{\mathbf{k}_{1}} \Gamma_{i \gamma, i / \gamma^{\prime}}^{(0) i_{2} \gamma_{2}, i_{1} \gamma_{1}}\left(-\mathbf{k},-\mathbf{k}_{1}, \mathbf{k}_{1}\right) \\
& \times\left[\widetilde{G}_{i_{1} \gamma_{1}, i_{2} \gamma_{2}}^{a a^{+}}\left(\mathbf{k}_{1}, \varepsilon_{1}\right)-\widetilde{G}_{i_{1} \gamma_{1}, i_{2} \gamma_{2}}^{a a^{+} *}\left(\mathbf{k}_{1}, \varepsilon_{1}\right)\right] \\
& \widetilde{\Sigma}_{\text {ee } i \gamma, i \prime \gamma \prime}^{(2)}(\mathbf{k}, \varepsilon)=\left(\frac{1}{2 \pi i}\right)^{2} \frac{1}{N^{2}} \int_{-\infty}^{\infty} d \varepsilon_{1} \int_{-\infty}^{\infty} d \varepsilon_{2} \\
& \times f\left(\varepsilon_{1}\right) f\left(\varepsilon_{2}\right) \sum_{\mathbf{k}_{1}, \mathbf{k}_{2}} \Gamma_{\mathrm{i}_{2} \gamma_{2}, i_{1} \gamma_{1}}^{(0) \mathrm{i} \gamma i_{3} \gamma_{3}}\left(-\mathbf{k},-\mathbf{k}_{1}-\mathbf{k}_{2}+\mathbf{k}, \mathbf{k}_{1}\right) \\
& \times\left\{\left[G_{i_{2} \gamma_{2}, i_{5} \gamma_{5}}^{a a^{+}}\left(\mathbf{k}-\mathbf{k}_{1}-\mathbf{k}_{2}, \varepsilon-\varepsilon_{1}-\varepsilon_{2}\right) G_{i_{1} \gamma_{1}, i_{4} \gamma_{4}}^{a a^{+} *}\left(\mathbf{k}_{1}, \varepsilon_{1}\right)-\right.\right. \\
& \left.-G_{i_{2} \gamma_{2}, i_{5} \gamma_{5}}^{a{ }^{+} *}\left(\mathbf{k}-\mathbf{k}_{1}-\mathbf{k}_{2}, \varepsilon-\varepsilon_{1}-\varepsilon_{2}\right) G_{i_{1} \gamma_{1}, i_{4} \gamma_{4}}^{a{ }^{+}}\left(\mathbf{k}_{1}, \varepsilon_{1}\right)\right] \\
& \times\left[\widetilde{G}_{i_{6} \gamma_{6}, i_{3} \gamma_{3}}^{a a^{+}}\left(\mathbf{k}_{2}, \varepsilon_{2}\right)-\widetilde{G}_{i_{6} \gamma_{6}, i_{3} \gamma_{3}}^{a{ }^{+} *}\left(\mathbf{k}_{2}, \varepsilon_{2}\right)\right] \\
& -\left[\widetilde{G}_{i_{2} \gamma_{2}, i_{5} \gamma_{5}}^{a a^{+}}\left(\mathbf{k}-\mathbf{k}_{1}-\mathbf{k}_{2}, \varepsilon-\varepsilon_{1}-\varepsilon_{2}\right)\right. \\
& \left.-\widetilde{G}_{i_{2} \gamma_{2}, i_{5} \gamma_{5}}^{a a^{+} *}\left(\mathbf{k}-\mathbf{k}_{1}-\mathbf{k}_{2}, \varepsilon-\varepsilon_{1}-\varepsilon_{2}\right)\right] \\
& \times\left[\widetilde{G}_{i_{1} \gamma_{1}, i_{4} \gamma_{4}}^{a \gamma^{+}}\left(\mathbf{k}_{1}, \varepsilon_{1}\right) \widetilde{G}_{i_{6} \gamma_{6}, i_{3} \gamma_{3}}^{a+}\left(\mathbf{k}_{2}, \varepsilon_{2}\right)\right. \\
& \left.\left.-\widetilde{G}_{i_{1} \gamma_{1}, i_{4} \gamma_{4}}^{a a^{+} *}\left(\mathbf{k}_{1}, \varepsilon_{1}\right) \widetilde{G}_{i_{6} \gamma_{6}, i_{3} \gamma_{3}}^{a a^{+} *}\left(\mathbf{k}_{2}, \varepsilon_{2}\right)\right]\right\} \\
& \times \widetilde{\Gamma}_{\mathrm{i}_{4} \gamma_{4}, i^{\prime} \gamma^{\prime}}^{\mathrm{i}_{5} \gamma_{5}, i_{6} \gamma_{6}}\left(\mathbf{k}_{1}+\mathbf{k}_{2}-\mathbf{k},-\mathbf{k}_{2}, \mathbf{k}_{1}\right) \text {. }
\end{aligned}
$$

The vertex part for the mass operator of electron-electron interplay is determined using the equation: 


$$
\begin{aligned}
& \widetilde{\Gamma}_{\mathrm{i}_{4} \gamma_{4}, i / \gamma^{\prime}}^{\mathrm{i}_{5} \gamma_{5}, i_{6} \gamma_{6}}\left(\mathbf{k}_{1}, \mathbf{k}_{2}, \mathbf{k}_{3}\right)=\Gamma_{\mathrm{i}_{4} \gamma_{4}, i \gamma^{\prime}}^{(0) \mathrm{i}_{5} \gamma_{5} i_{6} \gamma_{6}}\left(\mathbf{k}_{1}, \mathbf{k}_{2}, \mathbf{k}_{3}\right) \\
& -\frac{1}{2 \pi i} \frac{1}{N} \int_{-\infty}^{\infty} d \varepsilon f(\varepsilon) \sum_{\mathbf{k}_{4}} \Gamma_{\mathrm{i}_{4} \gamma_{4}, i_{8} \gamma_{8}}^{(0) \mathrm{i}_{5} \gamma_{5}, i_{7} \gamma_{7}}\left(\mathbf{k}_{1}, \mathbf{k}_{2}, \mathbf{k}_{4}\right) \\
& \times\left[\widetilde{G}_{i_{7} \gamma_{7}, i_{9} \gamma_{9}}^{a a^{+}}\left(\mathbf{k}_{4}, \varepsilon\right) \widetilde{G}_{i_{8} \gamma_{8}, i_{10} \gamma_{10}}^{a a^{+} *}\left(-\mathbf{k}_{1}-\mathbf{k}_{2}-\mathbf{k}_{4}, \varepsilon\right)\right. \\
& \left.-\widetilde{G}_{i_{7} \gamma_{7}, i_{9} \gamma_{9}}^{a a^{+} *}\left(\mathbf{k}_{4}, \varepsilon\right) \widetilde{G}_{i_{8} \gamma_{8}, i_{10} \gamma_{10}}^{a a^{+}}\left(-\mathbf{k}_{1}-\mathbf{k}_{2}-\mathbf{k}_{4}, \varepsilon\right)\right] \\
& \times \widetilde{\Gamma}_{\mathrm{i}_{10} \gamma_{10}, i / \gamma /}^{\mathrm{i}^{\prime} \gamma_{9}, i_{6} \gamma_{6}}\left(\mathbf{k}_{1}+\mathbf{k}_{2}+\mathbf{k}_{4},-\mathbf{k}_{4}, \mathbf{k}_{3}\right) \text {. }
\end{aligned}
$$

In Expression (81),

$$
\begin{aligned}
& \Gamma_{i_{1} \gamma_{1}, i \gamma}^{(0) i_{2} \gamma_{2}, i_{3} \gamma_{3}}\left(\mathbf{k}_{1}, \mathbf{k}_{2}, \mathbf{k}_{3}\right)= \\
& \sum_{1, n_{2}, n_{3}} \widetilde{v}_{n_{3} i_{3} \gamma_{3}, n i \gamma}^{(2)} n_{1} i_{1} \gamma_{1}, n_{2} i_{2} \gamma_{2} \exp \left(i \mathbf{k}_{1}\left(\mathbf{r}_{n_{1} i_{1}}-\mathbf{r}_{n i}\right)\right) \\
& \times \exp \left(i \mathbf{k}_{2}\left(\mathbf{r}_{n_{2} i_{2}}-\mathbf{r}_{n i}\right)+i \mathbf{k}_{3}\left(\mathbf{r}_{n_{3} i_{3}}-\mathbf{r}_{n i}\right)\right) \text {. }
\end{aligned}
$$

Cluster decomposition for the Green's function of electrons and phonons of disordered crystal may be derived from Equations (55)-(58). The density of electrons' and phonons' states are presented as an infinite series. Here, the procedure of scattering on clusters with different numbers of atoms are described by each term. It is shown that the contribution of the scattering procedure to electrons as well as phonons in clusters decreases with the increasing number of atoms in the cluster by a small parameter.

We have shown previously $[25,28,30]$ that the above-mentioned parameter stays small when many parameters of the system are changed, except possibly for narrow energy distance near the band edges.

By abandoning the input of activity of electron scattering at clusters containing three or even more atoms, which are small using the above-mentioned parameter for the density of electronic states, it is possible to obtain [28,29]:

$$
\begin{aligned}
& g_{e}(\varepsilon)=\frac{1}{v} \sum_{i, \delta, \sigma, \lambda, m_{\lambda i}} P_{0 i}^{\lambda m_{\lambda i}} g_{0 i \delta \sigma}^{\lambda m_{\lambda i}}(\varepsilon) \\
& g_{0 i \delta \sigma}^{\lambda m_{\lambda i}}(\varepsilon)=-\frac{1}{\pi} \operatorname{Im}\left\{\widetilde{G}+\widetilde{G} t^{\lambda m_{\lambda i} 0 i} \widetilde{G}+\sum_{(l j) \neq(0 i)} P_{l j 0 i}^{\lambda / m_{\lambda^{\prime} j} / \lambda m_{\lambda i}}\right. \\
& \left.\times \widetilde{G}\left[t^{\lambda^{\prime} m_{\lambda^{\prime} j} l j}+T^{(2) \lambda m_{\lambda i} 0 i, \lambda^{\prime} m_{\lambda^{\prime} j} l j}\right] \widetilde{G}\right\}^{0 i \delta \sigma, 0 i \delta \sigma} \\
& \lambda^{\prime}, m_{\lambda^{\prime} j} \\
& T^{(2) \lambda m_{\lambda i} 0 i, \lambda^{\prime} m_{\lambda^{\prime} j} l j}=\left[I-t^{\lambda m_{\lambda i} 0 i} \widetilde{G} t^{\lambda^{\prime} m_{\lambda^{\prime} j} l j} \widetilde{G}\right]^{-1} \\
& \times t^{\lambda m_{\lambda i} 0 i} \widetilde{G} t^{\lambda^{\prime} m_{\lambda^{\prime} j} l j}\left[I+\widetilde{G} t^{\lambda m_{\lambda i} 0 i}\right]
\end{aligned}
$$

Likewise, averaging for the phonon Green's function $G^{u u}(\varepsilon)$ produces the phononic density of states $[28,29]$

$$
\begin{gathered}
g_{p h}(\varepsilon)=\frac{1}{v} \sum_{i, \alpha, \lambda} P_{0 i g_{0 i \alpha}^{\lambda}}^{\lambda}(\varepsilon), \\
g_{0 i \alpha}^{\lambda}(\varepsilon)=-\frac{1}{\pi} 2 \frac{\varepsilon}{\hbar^{2}} M_{i} \operatorname{Im}\left\{\widetilde{G}+\widetilde{G} t^{\lambda 0 i} \widetilde{G}+\sum_{\substack{(l j) \underset{j}{\neq}(0 i) \\
\lambda^{\prime}}} P^{\lambda^{\prime} / \lambda} l j 0 i\right. \\
\times \widetilde{G}\left[t^{\lambda^{\prime} l j}+T^{\left.(2) \lambda 0 i, \lambda^{\prime} l j\right]} \widetilde{G}\right\}^{0 i \alpha, 0 i \alpha},
\end{gathered}
$$

where $\widetilde{G}=\widetilde{G}^{u u}(\varepsilon)$.

In Equation (84), $P^{\lambda / m_{\lambda^{\prime} j} / \lambda m_{\lambda i}}$ is the conditional probability to find the atom of type $\lambda^{\prime}$ in the site $(l j)$ containing the magnetic moment $m_{\lambda^{\prime} j^{\prime}}$, on the condition that hesitesin the unit 
cellinorigin ( $0 i$ ) have the atom of $\lambda$ type with the magnetic moment $m_{\lambda i} \cdot t_{n i}^{\lambda m_{\lambda i}}$ is the amount of the matrix constituent for the single-centre operator of scattering for the occasion where the atom of variety $\lambda$ is located at site (ni) and has a magnetic moment $m_{\lambda i}$ (seeRefs. [28,29]). This method for describing electron correlations in crystals is consistent with the approach described in Refs. [31,32].

For an ideally ordered crystal, the Green's function in Equation (55) is

$$
G(\varepsilon)=\lim \widetilde{G}(\varepsilon), \sigma(\varepsilon) \rightarrow 0,
$$

Where Green's function $\widetilde{G}(\varepsilon)$ is described using Equations (53) and (54). The energies of the electrons and phonons of the crystal are determined using the equations of the poles for the Green's functions

$$
\begin{gathered}
\operatorname{det}\left\|\varepsilon \delta_{i i \prime} \delta_{\gamma \gamma^{\prime}}-\widetilde{H}_{i \gamma, i \prime \gamma^{\prime}}(\mathbf{k}, \varepsilon)\right\|=0, \\
\operatorname{det}\left\|\frac{\varepsilon^{2}}{\hbar^{2}} M_{i} \delta_{i i \prime} \delta_{\alpha \alpha^{\prime}}-\widetilde{\Phi}_{i \alpha, i \prime \alpha \prime}(\mathbf{k}, \varepsilon)\right\|=0
\end{gathered}
$$

where $\widetilde{H}_{i \gamma, i \gamma \gamma^{\prime}}(\mathbf{k}, \varepsilon), \widetilde{\Phi}_{i \alpha, i \prime \alpha \prime}(\mathbf{k}, \varepsilon)$ is described by means of Equations (69) and (72).

\section{Energy Spectrum of Graphene including Adsorbed Potassium Atoms}

To calculate the electron spectrum of graphene with adsorbed potassium atoms, we chose the wave functions of the $2 \mathrm{~s}$ and $2 \mathrm{p}$ states of neutral non-interacting carbon atoms asthebasis. In the calculation of matrix elements of the Hamiltonian, we took three first coordination circles. The energy spectrum for graphene was computed employing the temperature $\mathrm{T}=0 \mathrm{~K}$. In our computations, we disrespect the re-normalisation of vertices of the mass operator of the electron-electron interplay. We consider the limiting case of an ordered arrangement of potassium atoms on the surface of graphene. The dependence of the electron energy on the wave vector is calculated, in this case, on the basis of the Equation (89) obtained in the work for the poles of Green's function.

In Figure 1, we exhibit the reliance of the electron energy $\varepsilon$ in graphene with adsorbed potassium atoms on the wave vector $k$. Thevector $\mathrm{k}$ is focused from the $\Gamma$-pointto the Dirac point, namely the K-point.

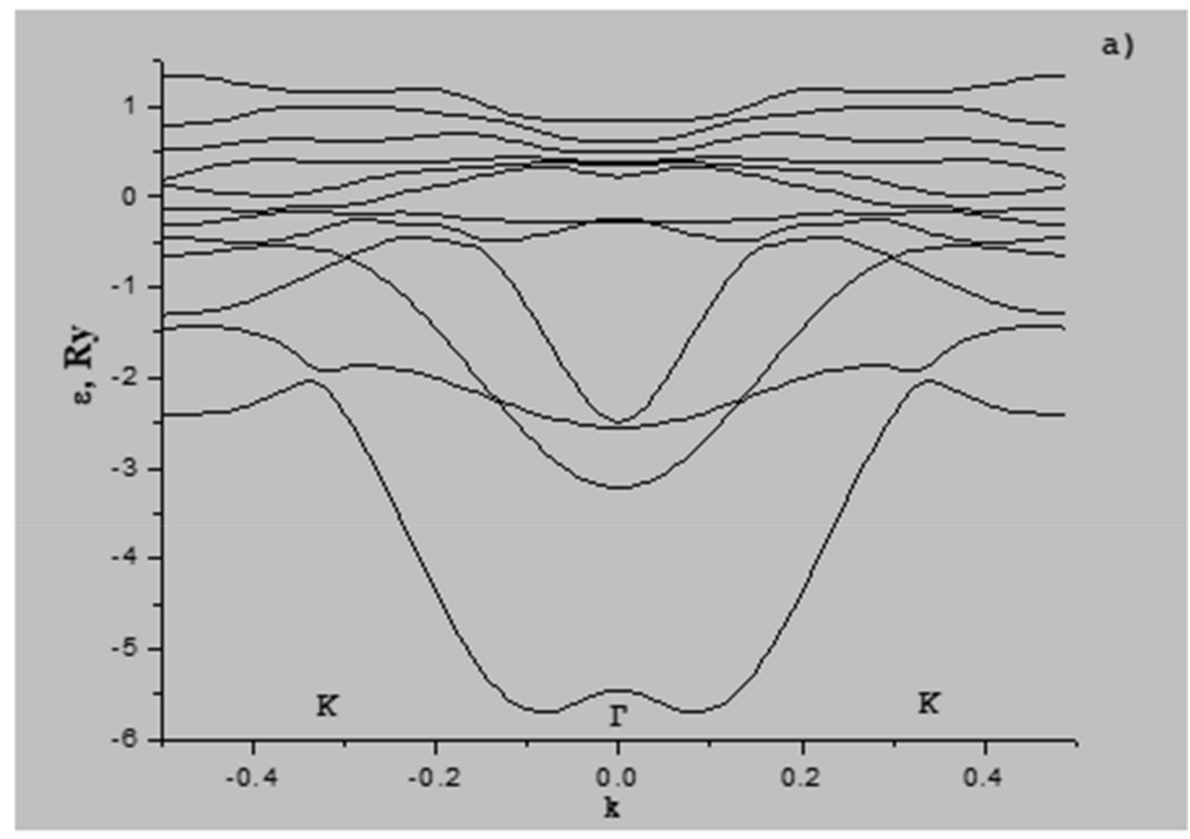

Figure 1. Cont. 


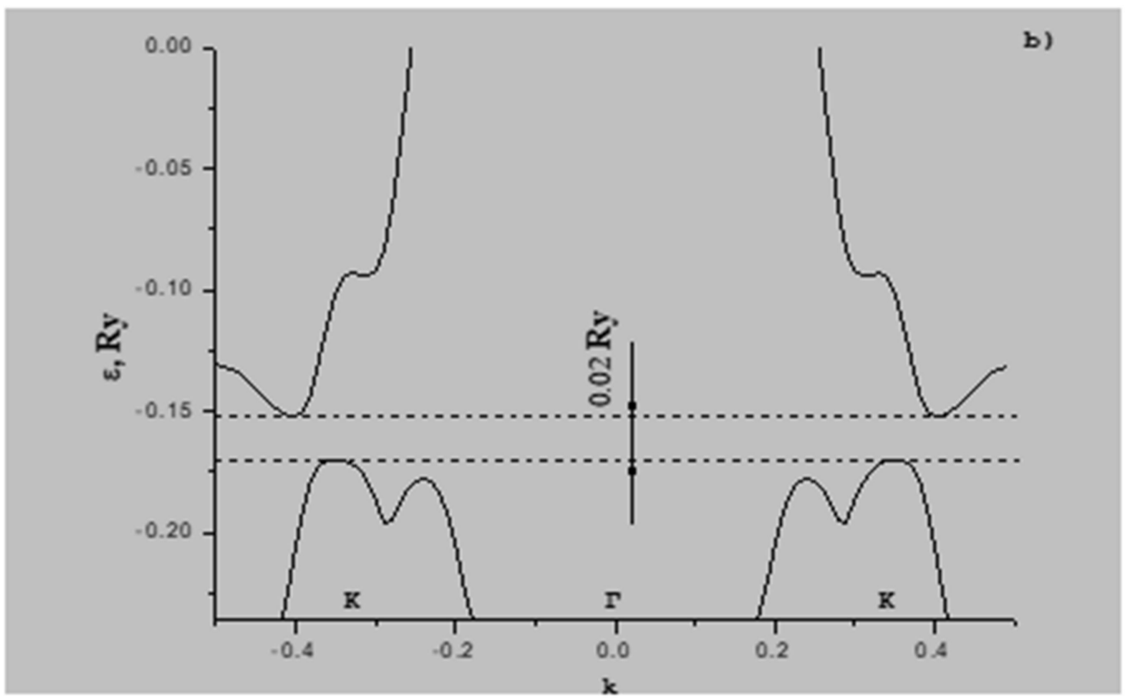

Figure 1. $(\mathbf{a}, \mathbf{b})$ Dependency for electron energy $\varepsilon$ at the wave vector $k$ in the graphene containing potassium impurity.

In Figure 1, the formative periodic separation from the potassium atom to the carbon atom is equal to $0.28 \mathrm{~nm}$. It is possible to see from Figure 1 that, in the system atisedadjustment of potassium atoms, the gap at the graphene energy spectrum emerges. Its numerical valueis dependenton the concentration of adsorbed potassium atoms, their position in the unit cell, as well as the distance to carbon atoms. We fixed that, at thealike potassium concentration, that the unit cell contains two carbon atoms and one potassium atom, where the potassium atom is placed on the graphene surface over a carbon atom at the separation of $0.286 \mathrm{~nm}$, and the energy gap is equal to $0.25 \mathrm{eV}$. The position of the Fermi level at the energy spectrum bank on the potassium concentration is at the energy distance $-0.36 \mathrm{Ry} \leq \varepsilon_{F} \leq R y 0.36$. Such a position occurs if the graphene is put on the potassium support.

\section{Conclusions}

Our contribution reveals an original method of characterisingthe electronic spectrum for thedisarrangedcrystals based on the Hamiltonian of electrons as well as phonons, and a diagram approach for the Green's function finding. Electronic states of the system werecharacterised using a multi-band, tight-binding approach. The Hamiltonian of the system was described using the wave functions of electrons at the atom nucleus field. The proposed approach containsa long-range Coulomb interplay of electrons located at different sites ofthe lattice. Exact formulations for Green's functions were derived using a diagram method. A system of equations for the spectrum of elementary excitations in an ideal crystal was obtained, in which the vertex components of the mass operators of electron-electron and electron-phonon interplays arerenormalised. Thismakes possible the performance of numerical computations of the energy spectrum for a system with a predetermined accuracy. In contrast to other approaches in which electron correlations areonly taken into account in the limiting cases of an infinitely large and infinitesimal electron density, in this method, electron correlations are described in the general case of an arbitrary density. Cluster increase is derived for the density of states of disorganisedsystems. We show that the addition of the electron scattering process to the clusters is decreasing across the augmenting number of places in the cluster, which is dependent on a small parameter. The calculation precision is decided bya small parameter of cluster enlargement of Green's function for electrons as well as phonons.

We have discovered that, at the potassium concentration equal to such that the unit cell contains two carbon atoms as well as one potassium atom, the potassium atom isplaced on the graphene surface upon thecarbon atom at the separation of $0.286 \mathrm{~nm}$, and the 
energy gap is equal to $0.25 \mathrm{eV} . S u c h$ an event happens ifthe graphene puton the potassium is supportive.

Author Contributions: Funding acquisition, S.P.K., R.I.E., and S.P.R.;Investigation, S.P.K., R.I.E., V.P.B., I.G.V., and S.P.R.; Writing-original draft, S.P.K., R.I.E., V.P.B., I.G.V., and S.P.R. All authors have read and agreed to the published version of the manuscript.

Funding: S.P.K. acknowledges support by the National Academy of Sciences of Ukraine (Project No.0116U002067)

Institutional Review Board Statement: Not applicable.

Informed Consent Statement: Not applicable.

Data Availability Statement: Not applicable.

Acknowledgments: Calculations were performed using Latvian Super Cluster (LASC), located in the Center of Excellence at Institute of Solid State Physics, the University of Latvia, which is supported by European Union Horizon 2020 Framework Programme H2020-WIDESPREAD-01-2016-2017-Teaming. Phase two under Grant Agreement No. 739508, project CAMART.

Conflicts of Interest: The authors declare no conflict of interest.

\section{References}

1. Harrison, W.A. Pseudopotentials in the Theory of Metals; Benjamin: New York, NY, USA, 1966.

2. Vanderbilt, D. Soft self-consistent pseudopotentials in a generalized eigenvalue formalism. Phys. Rev.B 1985, 41, 7892. [CrossRef] [PubMed]

3. Laasonen, K.; Car, R.; Lee, C.; Vanderbilt, D. Implementation of ultrasoft pseudopotentials in ab initio molecular dynamics. Phys. Rev. B 1991, 43, 6796. [CrossRef]

4. Blochl, P.E. Projector augmented-wave method. Phys. Rev. B 1994, 50, 17953. [CrossRef]

5. Kresse, G.; Joubert, D. From ultrasoft pseudopotentials to the projector augmented-wave method. Phys. Rev. B 1999, 59, 1758. [CrossRef]

6. Perdew, J.P.; Burke, K.; Ernzerhof, M. Generalized gradient approximation made simple. Phys. Rev. Lett. 1996, 77, 3865. [CrossRef] [PubMed]

7. Tao, J.; Perdew, J.P.; Staroverov, V.N.; Scuseria, G.E. Climbing the density functional ladder: Nonempirical meta-generalized gradient approximation designed for molecules and solids. Phys. Rev. Lett. 2003, 91, 146401. [CrossRef] [PubMed]

8. Perdew, J.P.; Kurth, S.; Zupan, A.; Blaha, P. Accurate density functional with correct formal properties: A step beyond the generalized gradient approximation. Phys. Rev. Lett. 1999, 82, 2544. [CrossRef]

9. Perdew, J.P.; Ruzsinszky, A.; Csonka, G.I.; Constantin, L.A.; Sun, J. Workhorse semilocal density functional for condensed matter physics and quantum chemistry. Phys. Rev. Lett. 2009, 103, 026403. [CrossRef]

10. Sun, J.; Marsman, M.; Csonka, G.I.; Ruzsinszky, A.; Hao, P.; Kim, Y.-S.; Kresse, G.; Perdew, J.P. Self-consistent meta-generalized gradient approximation within the projector-augmented-wave method. Phys. Rev. B 2011, 84, 035117. [CrossRef]

11. Kresse, G.; Furthmuller, J. Efficient iterative schemes for ab initio total-energy calculations using a plane-wave basis set. Phys. Rev. B 1996, 54, 11169. [CrossRef]

12. Frisch, M.J.; Trucks, G.W.; Schlegel, H.B. Gaussian 03, Revision C. 02 [CP]; Gaussian, Inc.: Wallingford, CT, USA, 2004.

13. Ivanovskaya, V.V.; Köhler, C.; Seifert, G. 3d metal nanowires and clusters inside carbon nanotubes: Structural, electronic, and magnetic properties. Phys. Rev. B 2007, 75, 075410. [CrossRef]

14. Porezag, D.; Frauenheim, T.; Köhler, T.; Seifert, G.; Kascher, R. Construction of tight-binding-like potentials on the basis of density-functional theory: Application to carbon. Phys. Rev. B 1995, 51, 2947. [CrossRef] [PubMed]

15. Elstner, M.; Porezag, D.; Jungnickel, G.; Elsner, J.; Haugk, M.; Frauenheim, T.; Suhai, S.; Seifert, G. Self-consistent-charge density-functional tight-binding method for simulations of complex materials properties. Phys. Rev. B 1998, 58, 7260. [CrossRef]

16. Köhler, C.; Seifert, G.; Gerstmann, U.; Elstner, M.; Overhof, H.; Frauenheim, T. Approximate density-functional calculations of spin densities in large molecular systems and complex solids. Phys. Chem. Chem. Phys. 2001, 3, 5109-5114. [CrossRef]

17. Ivanovskaya, V.V.; Seifert, G. Tubular structures of titanium disulfide TiS 2 . Solid State Commun. 2004, 130, 175. [CrossRef]

18. Ivanovskaya, V.; Heine, T.; Gemming, S.; Seifert, G. Structure, stability and electronic properties of composite Mo1-x Nbx S2 nanotubes. Phys. Status Solidi B 2006, 243, 1757. [CrossRef]

19. Enyaschin, A.; Gemming, S.; Heine, T.; Seifert, G.; Zhechkov, L. C28 fullerites-Structure, electronic properties and intercalates Phys. Chem. Chem. Phys. 2006, 8, 3320-3325. [CrossRef]

20. Slater, J.C.; Koster, G.F. Simplified LCAO method for the periodic potential problem. Phys. Rev. 1954, 94, 1498. [CrossRef]

21. Sharma, R.R. General expressions for reducing the Slater-Koster linear combination of atomic orbitals integrals to the two-center approximation. Phys. Rev. B 1979, 19, 2813. [CrossRef] 
22. Stocks, G.M.; Temmerman, W.M.; Gyorffy, B.L. Complete solution of the Korringa-Kohn-Rostoker coherent-potentialapproximation equations: Cu-Ni alloys. Phys. Rev. Lett. 1978, 41, 339. [CrossRef]

23. Stocks, G.M.; Winter, H. Self-consistent-field-Korringa-Kohn-Rostoker-coherent-potential approximation for random alloys. Z. Für Phys. B Condens. Matter 1982, 46, 95-98. [CrossRef]

24. Johnson, D.D.; Nicholson, D.M.; Pinski, F.J.; Gyorffy, B.L.; Stocks, G.M. Total-energy and pressure calculations for random substitutional alloys. Phys. Rev. B 1990, 41, 9701. [CrossRef] [PubMed]

25. Repetsky, S.P.; Shatnii, T.D. Thermodynamic Potential of a System of Electrons and Phonons in a Disordered Alloy. Theor. Math Phys. 2002, 131, 456. [CrossRef]

26. Abrikosov, A.A.; Gorkov, L.P.; Dzyaloshinski, I.E. Methods of Quantum Field Theory in Statistical Physics; Translated from the Russian and edited by Richard A. Silverman; Prentice-Hall: Englewood Cliffs, NJ, USA; Courier Corporation: Chelmsford, MA, USA, 1963.

27. Löwdin, P.O. On the Non-Orthogonality Problem Connected with the Use of Atomic Wave Functions in the Theory of Molecules and Crystals. J. Chem. Phys. 1950, 18, 365-375. [CrossRef]

28. Repetsky, S.; Vyshyvana, I.; Nakazawa, Y.; Kruchinin, S.; Bellucci, S. Electron transport in carbon nanotubes with adsorbed chromium impurities. Materials. 2019, 12, 524. [CrossRef]

29. Repetsky, S.; Vyshyvana, I.; Kruchinin, S.; Bellucci, S. Tight-binding model in the theory of disordered crystals. Mod. Phys. Lett. B 2020, 34, 2040065. [CrossRef]

30. Los', V.F.; Repetsky, S.P. A theory for the electrical conductivity of an ordered alloy. J. Phys. Condens. Matter 1994, 6, 1707. [CrossRef]

31. Kruchinin, S. Problems and Solutions in Special Relativity and Electromagnetism; World Scientific: Singapore, 2017 ; p. 140.

32. Kruchinin, S. Modern Aspect of Superconductivity: Theory of Superconductivity; World Scientific: Singapore, $2021 ;$ p. 308. 